\title{
Numerical Study of Astrophysics Equations by Meshless Collocation Method Based on Compactly Supported Radial Basis Function
}

\author{
K. Parand ${ }^{\mathrm{a}, *}$, M. Hemami ${ }^{\mathrm{a}}$ \\ ${ }^{a}$ Department of Computer Sciences, Shahid Beheshti University, G.C. Tehran 19697-64166, Iran
}

\begin{abstract}
In this paper, we propose compactly supported radial basis functions for solving some wellknown classes of astrophysics problems categorized as non-linear singular initial ordinary differential equations on a semi-infinite domain. To increase the convergence rate and to decrease the collocation points, we use the compactly supported radial basis function through the integral operations. Afterwards, some special cases of the equation are presented as test examples to show the reliability of the method. Then we compare the results of this work with some results and show that the new method is efficient and applicable.
\end{abstract}

Keywords: Lane-Emden type equation, Compact support radial basis functions, Isothermal Gas sphere, White Dwarf equation, Non-linear ordinary differential equation

\section{Introduction}

In recent decades, the so-called meshless methods have been extensively used to find approximate solutions of various types of linear and non-linear equations (Fasshauer, 2007) such as differential equations (DEs) and integral equations (IEs). Unlike the other methods which were used to mesh the domain of the problem, meshless methods don't require a structured grid and only make use of a scattered set of collocation points regardless of the connectivity information between the collocation points.

For the last years, the radial basis functions (RBFs) method was known as a powerful tool for the scattered data interpolation problem. One of the domain-type meshless methods is given in (Kansa, 1990a) in 1990, which directly collocates RBF, particularly the multiquadric (MQ) to find an approximate solution of linear and non-linear DEs. The RBFs can be compactly and globally supported. The global RBF's are infinitely differentiable and contain a free parameter $c$, called the shape parameter (Buhman, 2000; Islam and S. Haqb, 2009; Sara, 2005). The interested reader is referred to the recent books and paper by Buhmann (Buhman, 2000, 2004) and Wendland (Wendland, 2005) for more basic details about RBFs, compactly and globally supported and the convergence rate of the RBFs. In RBF method using globally RBFs, if $c$ increases, the system of equations to be solved becomes ill-conditioned. Cheng et al. (Cheng et al., 2003) showed that

\footnotetext{
${ }^{*}$ Corresponding author. Member of research group of Scientific Computing. Fax: +98 2122431653

Email addresses: k_parand@sbu. ac.ir (K. Parand), mohammadhemami@yahoo.com (M. Hemami) 
when $c$ is very large then the RBFs system error is of exponential convergence. To overcome the problems of globally RBF, alternative ways suggested such as domain decomposition(Lee and Hon, 2004), LU decomposition(Kansa and Hon, 2000), good matrix pre-conditioners(Kansa and Hon, 2000) change the global support RBFs with compactly support RBFs (CSRBFs)(Shen, 2012) which are local support and have not any parameter.

There are two basic approaches for obtaining basis functions from RBFs, namely direct approach (DRBF) based on differential process (Kansa, 1990b) and indirect approach (IRBF) based on an integration process (Mai-Duy, 2005; Mai-Duy and Tran-Cong, 2001a,b). Both approaches were tested on the solution of second order DEs and the indirect approach was found to be superior to the direct approach (Mai-Duy and Tran-Cong, 2001a).

In this paper, we use the indirect CSRBF (ICSRBF) for finding the solution of Lane-Emden type equations and also Isothermal gas sphere, White Dwarf equation.

This paper is arranged as following:

In Section 2 we describe the Lane-Emden equations. In Section 3 we survey several methods that have been used to solve Lane-Emden type equations. In Section 4, the properties of CSRBF and the way to construct the ICSRBF method for this type of equations are described. In Section 5 the proposed method is applied to some types of Lane-Emden equations, and a comparison is made with the existing solutions that were reported in other published works. Finally we give a brief conclusion in the last section.

\section{Lane-Emden type equations}

The Lane-Emden equation describes a variety of phenomena in theoretical physics and astrophysics, including aspects of stellar structure, the thermal history of a spherical cloud of gas, isothermal gas spheres, and thermionic currents.

Let $P(r)$ denote the total pressure at a distance $r$ from the center of spherical gas cloud. The total pressure is due to the usual gas pressure and a contribution from radiation:

$$
P=\frac{1}{3} \varsigma T^{4}+\frac{R T}{v},
$$

where $\varsigma, T, R$ and $v$ are respectively the radiation constant, the absolute temperature, the gas constant, and the specific volume(Agrawala and O'Regnab, 2007). Let $M(r)$ be the mass within a sphere of radius $r$ and $G$ the constant of gravitation. The equilibrium equations for the configuration are

$$
\begin{aligned}
& \frac{d P}{d r}=-\frac{G M(r)}{r^{2}}, \\
& \frac{d M(r)}{d r}=4 \pi \rho r^{2},
\end{aligned}
$$

where $\rho$ is the density, at a distance $r$ from the center of a spherical star.

Eliminating $M$ yields:

$$
\frac{1}{r^{2}} \frac{d}{d r}\left(\frac{r^{2}}{\rho} \frac{d P}{d r}\right)=-4 \pi G \rho
$$

Pressure and density $\rho=v^{-1}$ vary with $r$ and $P=K \rho^{1+\frac{1}{m}}$ where $K$ and $m$ are constants.

We can insert this relation into Eq. (1) for the hydrostatic equilibrium condition and from this 
rewrite equation to:

$$
\left[\frac{K(m+1)}{4 \pi G} \lambda^{\frac{1}{m}-1}\right] \frac{1}{r^{2}} \frac{d}{d r}\left(r^{2} \frac{d y}{d r}\right)=-y^{m}
$$

where $\lambda$ representing the central density of the star and $y$ is dimensionless quantity that are both related to $\rho$ through the following relation:

$$
\rho=\lambda y^{m},
$$

and let:

$$
\begin{aligned}
& r=a x, \\
& a=\left[\frac{K(m+1)}{4 \pi G} \lambda^{\frac{1}{m}-1}\right]^{\frac{1}{2}} .
\end{aligned}
$$

Inserting these relations into our previous relations we obtain the Lane-Emden equation (Dehghan and Saadatmandi, 2007):

$$
\frac{1}{x^{2}} \frac{d}{d x}\left(x^{2} \frac{d y}{d x}\right)=-y^{m}
$$

and with simplifying previous equation we have:

$$
y^{\prime \prime}(x)+\frac{2}{x} y^{\prime}(x)+y^{m}(x)=0, \quad x>0,
$$

with the boundary conditions:

$$
y(0)=1, \quad y^{\prime}(0)=0 .
$$

It has been claimed in the literature that only for $m=0,1$ and 5 the solutions of the Lane-Emden equation could be exact. For the other values of $m$, the Lane-Emden equation is to be integrated numerically. In this paper, we solve it for $m=1,1.5,2,2.5,3,4$ and 5 .

\section{Methods have been proposed to solve Lane-Emden type equation}

in years, many analytical and numerical methods have been used to solve Lane-Emden equations, the main difficulty arises in the singularity of the equations at $x=0$. Currently, most techniques which were used in handling the Lane-Emden type problems are based on either series solutions or perturbation techniques.

Bender et al. (Bender et al., 1989), proposed a new perturbation technique based on an artificial parameter $\delta$, the method is often called $\delta$-method.

Mendelzweig and Tabakin (Mendelzweig and Tabakin, 2001) used quasi-linearization approach to solve the standard Lane-Emden equation. This method approximates the solution of a non-linear differential equation by treating the non-linear terms as a perturbation about the linear ones, and unlike perturbation theories is not based on the existence of some small parameter. He showed that the quasi-linearization method gives excellent results when applied to different no-nlinear ordinary differential equations in physics, such as the Blasius, Duffing, Lane-Emden and Thomas-Fermi equations.

Shawagfeh (Shawagfeh, 1993) applied a non-perturbative approximate analytical solution for the Lane-Emden type equation using the Adomian decomposition method. His solution was in 
the form of a power series. He used Padé approximants method to accelerate the convergence of the power series.

In (Wazwaz, 2001), Wazwaz employed the Adomian decomposition method with an alternate framework designed to overcome the difficulty of the singular point. It was applied to the differential equations of Lane-Emden type. In future (Wazwaz, 2006) he used the modified decomposition method for solving analytical treatment of non-linear differential equations such as Lane-Emden equations. The modified method accelerates the rapid convergence of the series solutions, dramatically reduces the size of work, and provides the solution by using few iterations only without any need to the so-called Adomian polynomials.

Liao (Liao, 2003) provided a reliable, easy-to-use analytical algorithm for Lane-Emden type equations. This algorithm logically contained the well-known Adomian decompositions method. Different from all other analytical techniques, this algorithm itself provides us with a convenient way to adjust convergence regions even without Padé technique.

$\mathrm{He}(\mathrm{He}, 2003)$ employed Ritz's method to obtain an analytical solution of the problem. By the semi-inverse method, a variational principle is obtained for the Lane-Emden equation, which he gave much numerical convenience when applied to finite element methods or the Ritz method.

Parand et al. (Parand and Khaleqi, 2016; Parand and Razzaghi, 2004a,b) presented some numerical techniques to solve higher ordinary differential equations such as Lane-Emden. Their approach was based on a rational Chebyshev and rational Legendre tau method. They presented the derivative and product operational matrices of rational Chebyshev and rational Legendre functions.

These matrices together with the tau method were utilized to reduce the solution of these physical problems to the solutions of systems of algebraic equations. Also, in some paper's (Parand et al., 2011a,b, 2010a, 2013b), Parand et al. applied Hermite function collocation method (HFC), Bessel function collocation method and meshless collocation method based on Radial basis function (RBFs) to solving the Lane-Emden type equations.

Ramos (Ramos, 2003, 2005, 2007, 2008) solved Lane-Emden equation through different methods. In (Ramos, 2005) he presented linearzation methods for singular initial value problems in second order ordinary differential equation such as Lane-Emden. These methods result in linear constant-coefficient ordinary differential equations which can be integrated analytically, thus they yield piecewise analytical solutions and globally smooth solutions (Ramos, 2007). Later, he developed piecewise-adaptive decomposition methods for the solution of non-linear ordinary differential equations. Piecewise-decomposition methods provide series solutions in intervals which are subject to continuity conditions at the end points of each interval and their adoption is based on the use of either a fixed number of approximants and a variable step size, a variable number of approximants and a fixed step size or a variable number of approximants and a variable step size. In (Ramos, 2008), series solutions of the Lane-Emden equation, based on either a volterra integral equation formulation or the expansion of the dependent variable in the original ordinary differential equation are presented and compared with series solutions obtained by means of integral or differential equations based on a transformation of the dependent variables.

Yousefi (Yousefi, 2006) used integral operator and converted Lane-Emden equations to integral equations and then applied Legendre Wavelet approximations. In this work properties of Legendre wavelet together with the Gaussian integration method were utilized to reduce the integral equations to the solution of algebraic equations. By his method, the equation was formulated on $[0,1]$.

Chowdhury and Hashim (Chowdhury and Hashim, 2009) obtained analytical solutions of the 
generalized Emden-Fowler type equations in the second order ordinary differential equations by homotopy-perturbation method (HPM). This method is a coupling of the perturbation method and the homotopy method. The main feature of the HPM (Dehghan and Shakeri, 2008b) is that it deforms a difficult problem into a set of problems which are easier to solve. HPM yields solution in convergent series forms with easily computable terms.

Aslanov (Aslanov, 2008) constructed a recurrence relation for the components of the approximate solution and investigated the convergence conditions for the Emden-Fowler type of equations. He improved the previous results on the convergence radius of the series solution.

Dehghan and Shakeri (Dehghan and Shakeri, 2008a) investigated Lane-Emden equation using the variational iteration method and showed the efficiency and applicability of their procedure for solving this equation. Their technique does not require any discretization, linearization or small perturbations and therefore reduces the volume of computations.

Bataineh et al. (Bataineh et al., 2009) obtained analytical solutions of singular initial value problems (IVPs) of the Emden-Fowler type by the homotopy analysis method (HAM). Their solutions contained an auxiliary parameter which provided a convenient way of controlling the convergence region of the series solutions. It was shown that the solutions obtained by the Adomian decomposition method (ADM) and the homotopy-perturbation method (HPM) are only special cases of the HAM solutions.

singh et al. (Singh et al., 2009) used the modified Homotopy analysis method for solving the Lane-Emden-equation and White Dwarf equation.

Adibi and Rismani in (Adibi and Rismani, 2010) proposed the approximate solutions of singular IVPs of the Lane-Emden type in second-order ordinary differential equations by improved Legendre-spectral method. The Legendre-Gauss point used as collocation nodes and Lagrange interpolation is employed in the Volterra term.

Karimi vanani and Aminataei (Vanani and Aminataei, 2010) provide a numerical method which produces an approximate polynomial solution for solving Lane-Emden equations as singular initial value problem. They are first, used as an integral operator and convert Lane-Emden equations into integral equations, then convert the acquired integral equation into a power series and finally, they transforming the power series into pade series form.

Kaur et al. (Kaur et al., 2013), obtained the Haar wavelet approximate solutions for the generalized Lane-Emden equations. This method was based on the quasi-linearization approximation and replacement of an unknown function through a truncated series of Haar wavelet series of the function.

Other researchers try to solve the Lane-Emden type equations with several methods, For example, Yıldırım and Öziş (Yıldırım and Öziş, 2007, 2009) by using HPM and VIM methods, Benko et al. (Benko et al., 2008) by using Nyström method, Iqbal and javad (Iqbal and Javad, 2011) by using Optimal HAM, Boubaker and Van Gorder (Boubaker and Van-Gorder, 2012) by using Boubaker polynomials expansion scheme, Daşcioğlu and Yaslan (Akyüz-Daşcioğlu and Çerdik Yaslan, 2011) by using Chebyshev collocation method, Yüzbaşı (Yüzbaşı, 2011; Yüzbaş1 and Sezer, 2013) by using Bessel matrix and improved Bessel collocation method, Boyd (Boyd, 2011) by using Chebyshev spectral method, Bharwy and Alofi (Bharwy and Alofi, 2012) by using Jacobi-Gauss collocation method, Pandey et al. (Pandey and Kumar, 2012; Pandey et al., 2012) by using Legendre and Brenstein operation matrix, Rismani and monfared (Rismani and Monfared, 2012) by using Modified Legendre spectral method, Nazari-Golshan et al. (Nazari-Golshan et al., 2013) by using Homotopy perturbation with Fourier transform, Doha et al. (Doha et al., 2013) by using second kind Chebyshev operation matrix algorithm, Carunto and bota (Caruntu and Bota, 2013) by using Squared reminder minimization method, Mall and 
Chakaraverty (Mall and Chakraverty, 2014) by using Chebyshev Neural Network based model, Gürbüz and sezer (Gürbüz and Sezer, 2014) by using Laguerre polynomial, Kazemi-Nasab et al. (Kazemi-Nasab et al., 2015) by using Chebyshev wavelet finite difference method, Hosseini and Abbasbandy (Hosseini and Abbasbandy, 2015) by using combination of spectral method and ADM method and Azarnavid et al. (Azarnavid et al., 2015) by using Picard-Reproducing Kernel Hilbert Space Method

\section{ICSRBF method}

\section{1. $C S R B F$}

Many problems in science and engineering arise in infinite and semi-infinite domains. Different numerical methods have been proposed for solving problems on various domains such as FEM(Bu et al., 2015; Choi and Kweon, 2016), FDM(Bu et al., 2015; Noye and Dehghan, 1999), Spectral(Parand et al., 2013a, 2010b, 2016, 2013b; Rad et al., 2014) methods and meshfree methods(Abbasbandy et al., 2014; Dehghan and Shokri, 2009a; Rad et al., 2012; Shokri and Dehghan, 2010). The use of the RBF is one of the popular meshfree method for solving the differential equations (Dehghan and Shokri, 2008, 2009c; Rad et al., 2015a,b). For many years the global radial basis functions such as Gaussian, Multi quadric, Thin plate spline, Inverse multiqudric and others were used (Dehghan and Shokri, 2009b; Parand and Rad, 2013; Rashidi et al., 2014) to solve different DEs and interpolation. These functions are globally supported and generate a system of equations with ill-condition full matrix. To convert the ill-condition matrix to a wellcondition matrix, CSRBFs can be used instead of global RBFs. CSRBFs can convert the global scheme into a local one with banded matrices, Which makes the RBF method more feasible for solving large-scale problem (Wong et al., 2002).

\section{Wendland's functions}

The most popular family of CSRBF are Wendland functions. These functions were introduced by Holger Wendland in 1995 (Wendland, 1995). he started with the truncated power function $\phi_{l}(r)=(1-r)_{+}^{l}$ which is strictly positive definite and radial on $\mathbb{R}^{s}$ for $l \geq\left\lfloor\frac{s}{2}\right\rfloor+1$, and then he walks through dimensions by repeatedly applying the monté operator (I).

Definition 1 (Fasshauer, 2007) with $\phi_{l}(r)=(1-r)_{+}^{l}$ he defines

$$
\phi_{s, k}=I^{k} \phi_{\left\lfloor\frac{s}{2}\right\rfloor+k+1}
$$

it turns out that the functions $\phi_{s, k}$ are all supported on $[0,1]$.

Theorem 1 (Fasshauer, 2007) The functions $\phi_{s, k}$ are strictly positive definite (SPD) and radial on $\mathbb{R}^{s}$ and are of the form

$$
\phi_{s, k}(r)= \begin{cases}p_{s, k}(r) & r \in[0,1], \\ 0 & r>1,\end{cases}
$$

with a univariate polynomial $p_{s, k}$ of degree $\left\lfloor\frac{s}{2}\right\rfloor+3 k+1$. Moreover, $\phi_{s, k} \in C^{2 k}(R)$ are unique up to a constant factor, and the polynomial degree is minimal for given space dimension $s$ and smoothness $2 k$ (Fasshauer, 2007). Wendland gave recursive formulas for the functions $\phi_{s, k}$ for all $s, k$. 
Theorem 2 (Fasshauer, 2007) The functions $\phi_{s, k}, k=0,1,2,3$, have form

$$
\begin{aligned}
& \phi_{s, 0}=(1-r)_{+}^{l}, \\
& \phi_{s, 1} \doteq(1-r)_{+}^{l+1}[(l+1) r+1], \\
& \phi_{s, 2} \doteq(1-r)_{+}^{l+2}\left[\left(l^{2}+4 l+3\right) r^{2}+(3 l+6) r+3\right], \\
& \phi_{s, 3} \doteq(1-r)_{+}^{l+3}\left[\left(l^{3}+9 l^{2}+23 l+15\right) r^{3}+\left(6 l^{2}+36 l+45\right) r^{2}\right. \\
& +(15 l+45) r+15],
\end{aligned}
$$

where $l=\left\lfloor\frac{s}{2}\right\rfloor+k+1$, and the symbol $\doteq$ denotes equality up to a multiplicative positive constant. The case $k=0$ directly follows from the definition 1 . application of the definition 1 for the case $k=1$ yields

$$
\begin{aligned}
& \phi_{s, 1}(r)=\left(I \phi_{l}\right)(r)=\int_{r}^{\infty} t \phi_{l}(t) d t \\
& =\int_{r}^{\infty} t(1-t)_{+}^{l} d t=\int_{r}^{1} t(1-t)^{l} d t \\
& =\frac{1}{(l+1)(l+2)}(1-r)^{l+1}[(l+1) r+1],
\end{aligned}
$$

where the compact support of $\phi_{l}$ reduces the improper integral to a definite integral which can be evaluated using integration by parts. The other two cases are obtained similarly by repeated application of $I$.(Fasshauer, 2007) We showed most of the wendland functions in table 1 .

Table 1: Wendland's compactly supported radial function for various choices of $\mathrm{k}$ and $\mathrm{s}=3$.

\begin{tabular}{lll}
\hline$\phi_{s, k}$ & smoothness & SPD \\
\hline$\phi_{3,0}(r)=(1-r)_{+}^{2}$ & $C^{0}$ & $\mathbb{R}^{3}$ \\
$\phi_{3,1}(r) \doteq(1-r)_{+}^{4}(4 r+1)$ & $C^{2}$ & $\mathbb{R}^{3}$ \\
$\phi_{3,2}(r) \doteq(1-r)_{+}^{6}\left(35 r^{2}+18 r+3\right)$ & $C^{4}$ & $\mathbb{R}^{3}$ \\
$\phi_{3,3}(r) \doteq(1-r)_{+}^{8}\left(32 r^{3}+25 r^{2}+8 r+1\right)$ & $C^{6}$ & $\mathbb{R}^{3}$ \\
$\phi_{3,4}(r) \doteq(1-r)_{+}^{10}\left(429 r^{4}+450 r^{3}+210 r^{2}+50 r+5\right)$ & $C^{8}$ & $\mathbb{R}^{3}$ \\
$\phi_{3,5}(r) \doteq(1-r)_{+}^{12}\left(2048 r^{5}+2697 r^{4}+1644 r^{3}+566 r^{2}+108 r+9\right)$ & $C^{10}$ & $\mathbb{R}^{3}$ \\
\hline
\end{tabular}

\subsection{Interpolation by CSRBFs}

To interpolate or approximate one dimentional function $y(x)$, we can represent it by a CSRBF as

$$
y(x) \approx y_{n}(x)=\sum_{i=1}^{N} \xi_{i} \phi_{i}(x)=\Phi^{T}(x) \Xi,
$$

where

$$
\begin{aligned}
& \phi_{i}(x)=\phi\left(\frac{\left\|x-x_{i}\right\|}{r_{\omega}}\right), \\
& \Phi^{T}(x)=\left[\phi_{1}(x), \phi_{2}(x), \cdots, \phi_{N}(x)\right], \\
& \Xi=\left[\xi_{1}, \xi_{2}, \cdots, \xi_{N}\right]^{T}, \\
& 7
\end{aligned}
$$


(3)

$x$ is the input, $r_{\omega}$ is the local support domain and $\xi_{i}$ s are the set of coefficients to be determined. By using the local support domain, we mapped the domain of problem to CSRBF local domain. By choosing $N$ interpolate points $\left(x_{j}, j=1,2, \cdots, N\right)$ in domain:

$$
y_{j}=\sum_{i=1}^{N} \xi_{i} \phi_{i}\left(x_{j}\right)(j=1,2, \cdots, N) .
$$

To summarize the discussion on the coefficients matrix, we define

$$
A \Xi=Y,
$$

where :

$$
\begin{aligned}
& Y=\left[y_{1}, y_{2}, \cdots, y_{N}\right]^{T}, \\
& A=\left[\Phi^{T}\left(x_{1}\right), \Phi^{T}\left(x_{2}\right), \cdots, \Phi^{T}\left(x_{N}\right)\right]^{T} \\
& =\left(\begin{array}{cccc}
\phi_{1}\left(x_{1}\right) & \phi_{2}\left(x_{1}\right) & \cdots & \phi_{N}\left(x_{1}\right) \\
\phi_{1}\left(x_{2}\right) & \phi_{2}\left(x_{2}\right) & \cdots & \phi_{N}\left(x_{2}\right) \\
\vdots & \vdots & \ddots & \vdots \\
\phi_{1}\left(x_{N}\right) & \phi_{2}\left(x_{N}\right) & \cdots & \phi_{N}\left(x_{N}\right)
\end{array}\right) .
\end{aligned}
$$

Note that $\phi_{i}\left(x_{j}\right)=\phi\left(\frac{\left\|x_{i}-x_{j}\right\|}{r_{\omega}}\right)$, by solving the system (4), the unknown coefficients $\xi_{i}$ will be achieved.

\subsection{ICSRBF method}

In this paper, we construct the $\phi_{i}(x)$ in Eq. (5) by using the Wendland function with parameter $k=3$ and $l=5$.

At first, we approximate the highest order derivative in the problem by expansion of CSRBFs:

$$
\frac{d^{2}}{d x^{2}} y(x) \simeq y_{n}^{(2)}(x)=\sum_{i=1}^{N} \xi_{i} \phi_{i}(x),
$$

where $\phi_{i}(x)$ is the CSRBF and $r_{\omega}$ is the local support domain and coefficients $\xi_{i}$ must be determined. Now we define the lower order of derivatives and unknown function $y(x)$ using GaussLegendre integration as follows:

$$
\begin{aligned}
& \frac{d}{d x} y(x) \simeq \int_{0}^{x} y_{n}^{(2)}(t) d t-\left.\frac{d}{d x} y(x)\right|_{x=0}=\frac{x}{2} \sum_{j=1}^{q} \sum_{i=1}^{N} w[j] \xi_{i} \phi_{i}\left(\frac{x}{2} \eta[j]+\frac{x}{2}\right)=\left(y_{n}^{(1)}(x)\right), \\
& y(x) \simeq \int_{0}^{x}\left(y_{n}^{(1)}(t)\right) d t-y(0)=\left(y_{n}(x)\right),
\end{aligned}
$$

where $w[j]$ are weighted coefficients of Gauss-Legendre integration and define as follow:

$$
w[j]=\frac{2}{\left.\left(1-\eta[j]^{2}\right)\left(\frac{d^{2}}{d x^{2}} P_{q}(x)\right)\right|_{x=\eta[j]}},
$$


$P_{q}(x)$ is Legendre polynomial of order $q$ and $\eta[j]$ is $j$-th root of $P_{q-1}(x)$.

To satisfy the initial conditions of problem, we have:

$$
\begin{aligned}
& \left(y_{n}^{(1)}(x)\right)=\int_{0}^{x} y_{n}^{(2)}(t) d t, \\
& \left(y_{n}(x)\right)=\int_{0}^{x}\left(y_{n}^{(1)}(t)\right) d t+1,
\end{aligned}
$$

then, we produce the residual function as follows:

$$
\operatorname{Res}(x)=x y_{n}^{(2)}(x)+\alpha\left(y_{n}^{(1)}(x)\right)+x\left(y_{n}(x)\right)^{m},
$$

now, obtain $N$-nodes as follows:

$$
x_{j}=L\left(\frac{i}{N}\right)^{\gamma}, \quad j=1,2, \cdots, N,
$$

where $L$ is the last collocate node in domain and $\gamma$ is arbitrary parameter. For Lane-Emden type equations, we selected $\gamma$ between 1.5 and 1.8 , because with these values we found the best $\|$ Res $\|_{2}$ for solution equations.

By using $x_{j}, j=1,2, \ldots, N$ and residual function, we obtain $N$ equation, so by solving these equations we obtain the unknown coefficients $\xi_{i=1}^{N}$.

The result of this section can be summarized in the following algorithm for the IVP:

$$
F\left(x, y(x), y^{\prime}(x), y^{\prime \prime}(x)\right)=0, \quad y(0)=a, \quad y^{\prime}(0)=b .
$$

Algorithm The algorithm works in the following manner:

(1) Choose $N$ center points $\left\{X_{j}\right\}_{j=1}^{N}$ from domain $[0, \infty)$.

(2) Approximate $y^{\prime \prime}(x)$ as the from $u_{N}^{\prime \prime}(x)=\sum_{i=1}^{N} \xi_{i} \phi_{i}(x)$.

(3) Obtain $y^{\prime}(x)$ by using defined integral operation $I_{\chi}(h(x))=\int_{0}^{x} h(t) d t$ in the form $u_{N}^{\prime}(x)=$ $\sum_{i=1}^{N} \xi_{i} \int_{0}^{x} \phi_{i}(t) d t+b$.

(4) Obtain $y(x)$ by using defined integral operation $I_{\chi}(h(x))=\int_{0}^{x} h(t) d t$ in the form $u_{N}(x)=$ $\int_{0}^{x} u_{N}^{\prime}(t) d t+a$.

(4) Substitiute $u_{N}(x), u_{N}^{\prime}(x)$ and $u_{N}^{\prime \prime}(x)$ into the main problem and create residual function $\operatorname{Res}(\mathrm{x})$.

(5) Substitiute collocation points $\left\{X_{j}\right\}_{j=1}^{N}$ into the $\operatorname{Res}(\mathrm{x})$.

(6) Solve the $N$ equations with $N$ unknown coefficients $\left\{\xi_{i}\right\}_{i=1}^{N}$ and find the numerical solution.

$$
\operatorname{Res}\left(x_{j}\right)=0, \quad j=1,2, \cdots, N .
$$

\section{Application}

In this section, we apply ICSRBF method to solve the Lane-Emden type equations. In general the Lane-Emden type equation are formulated as follows:

$$
y^{\prime \prime}(x)+\frac{\alpha}{x} y^{\prime}(x)+p(x) q(y(x))=h(x), \quad \alpha x \geq 0,
$$

with initial conditions :

$$
y(0)=A, \quad y^{\prime}(0)=B,
$$


where $\alpha, A$ and $B$ are real constants and $p(x), q(y(x))$ and $h(x)$ are some given function. To apply the collocation method, we construct the residual function by substituting $y_{n}(x)$ in the Lane-Emden type Eq. (13):

$$
\operatorname{Res}(x)=y_{n}^{(2)}(x)+\frac{\alpha}{x} y_{n}^{(2)}(x)+p(x) q\left(y_{n}(x)\right)-h(x) .
$$

The equation for obtaining the coefficient $\xi_{i} s$ arise from equalizing $\operatorname{Res}(x)$ to zero at $N$ point (11):

$$
\operatorname{Res}\left(x_{j}\right)=0, \quad j=0,1,2, \ldots, N .
$$

By solving this set of equations we obtain a approximate function $y_{n}(x)$. Note that these $N$ equations generate a set of $N$ nonlinear equations which can be solved by a well-known method such as Newton method for unknown coefficient $\xi_{i} s$.

\subsection{Example 1 (The standard Lane-Emden equation)}

Table 2: Comparison of the first zeros of standard Lane Emden equations between the present method and exact numerical value given by Horedt(Horedt, 2004).

\begin{tabular}{lllllll}
\hline $\mathrm{m}$ & $\mathrm{N}$ & $r_{\omega}$ & $\mathrm{L}$ & The present method & Horedt & Error \\
\hline 0 & 40 & 6.5 & 10 & 2.44948974 & 2.44948974 & $0.00 \mathrm{e}-00$ \\
1 & 40 & 6.5 & 10 & 3.14159265 & 3.14159265 & $0.00 \mathrm{e}-00$ \\
1.5 & 30 & 2 & 4 & 3.65375388 & 3.65375374 & $1.40 \mathrm{e}-07$ \\
2 & 20 & 4 & 6 & 4.35287462 & 4.35287460 & $2.00 \mathrm{e}-08$ \\
2.5 & 20 & 4 & 6 & 5.35527531 & 5.35527546 & $1.50 \mathrm{e}-07$ \\
3 & 20 & 6.5 & 8 & 6.89684855 & 6.89684862 & $7.00 \mathrm{e}-08$ \\
4 & 20 & 14 & 16 & 14.9715808 & 14.9715463 & $3.45 \mathrm{e}-05$ \\
\hline
\end{tabular}

Table 3: Comparison of $y(x)$ of standard Lane-Emden equation between present method and exact values given by Horedt(Horedt, 2004), for $m=2$.

\begin{tabular}{llll}
\hline $\mathrm{x}$ & ICSRBF & Horedt & Error \\
\hline 0.00 & 1.0000000 & 1.0000000 & $0.00 \mathrm{e}+00$ \\
0.1 & 0.9983350 & 0.9983349 & $0.00 \mathrm{e}+00$ \\
0.5 & 0.9593527 & 0.9593527 & $0.00 \mathrm{e}+00$ \\
1.0 & 0.8486541 & 0.8486541 & $0.00 \mathrm{e}+00$ \\
3.0 & 0.2418240 & 0.2418241 & $1.00 \mathrm{e}-07$ \\
4.0 & 0.0488398 & 0.0488401 & $3.00 \mathrm{e}-07$ \\
4.3 & 0.0068107 & 0.0068109 & $2.00 \mathrm{e}-07$ \\
4.35 & 0.0003660 & 0.0003660 & $0.00 \mathrm{e}+00$ \\
\hline
\end{tabular}

For $p(x)=1, q(y(x))=y^{m}(x), \alpha=2, A=1, h(x)=0$, and $B=0$, Eq. (13) is the standard Lane-Emden eqution that was used to model the thermal behaviour of a spherical cloud 
Table 4: Comparison of $y(x)$ of standard Lane-Emden equation between present method and exact values given by Horedt(Horedt, 2004), for $m=3$.

\begin{tabular}{llll}
$\mathrm{x}$ & ICSRBF & Horedt & Error \\
\hline 0.00 & 1.0000000 & 1.0000000 & $0.00 \mathrm{e}+00$ \\
0.1 & 0.9983358 & 0.9983358 & $0.00 \mathrm{e}+00$ \\
0.5 & 0.9598391 & 0.9598391 & $0.00 \mathrm{e}+00$ \\
1.0 & 0.8550575 & 0.8550576 & $1.00 \mathrm{e}-07$ \\
5.0 & 0.1108198 & 0.1108198 & $0.00 \mathrm{e}+00$ \\
6.0 & 0.0437379 & 0.0437380 & $1.00 \mathrm{e}-07$ \\
6.8 & 0.0041677 & 0.0041678 & $1.00 \mathrm{e}-07$ \\
\hline
\end{tabular}

Table 5: Comparison of $y(x)$ of standard Lane-Emden equation between present method and exact values given by Horedt(Horedt, 2004), for $m=4$.

\begin{tabular}{llll}
$\mathrm{x}$ & ICSRBF & Horedt & Error \\
\hline 0.00 & 1.0000000 & 1.0000000 & $0.00 \mathrm{e}+00$ \\
0.1 & 0.9983401 & 0.9983367 & $3.40 \mathrm{e}-06$ \\
0.2 & 0.9933921 & 0.9933862 & $5.90 \mathrm{e}-06$ \\
0.5 & 0.9603107 & 0.9603109 & $2.00 \mathrm{e}-07$ \\
1.0 & 0.8608195 & 0.8608138 & $5.70 \mathrm{e}-06$ \\
5.0 & 0.2359598 & 0.2359227 & $3.71 \mathrm{e}-06$ \\
10 & 0.05965343 & 0.0596727 & $1.92 \mathrm{e}-05$ \\
14 & 0.0083590 & 0.0083305 & $2.85 \mathrm{e}-05$ \\
14.9 & 0.00058404 & 0.0005764 & $7.64 \mathrm{e}-06$ \\
\hline
\end{tabular}

Table 6: $\|$ Res $\|_{2}$ of the standard Lane-Emden equations for $m=1.5,2,2.5,3,4$ respectively.

\begin{tabular}{llllll}
\hline $\mathrm{n}$ & $\mathrm{m}=1.5$ & $\mathrm{~m}=2$ & $\mathrm{~m}=2.5$ & $\mathrm{~m}=3$ & $\mathrm{~m}=4$ \\
\hline 5 & $5.516 \mathrm{e}-03$ & $1.434 \mathrm{e}-02$ & $1.338 \mathrm{e}-03$ & $9.996 \mathrm{e}-02$ & $1.869 \mathrm{e}-00$ \\
10 & $7.250 \mathrm{e}-05$ & $1.646 \mathrm{e}-04$ & $7.148 \mathrm{e}-06$ & $1.851 \mathrm{e}-04$ & $6.436 \mathrm{e}-03$ \\
15 & $1.050 \mathrm{e}-06$ & $1.379 \mathrm{e}-05$ & $5.919 \mathrm{e}-09$ & $1.699 \mathrm{e}-08$ & $5.362 \mathrm{e}-03$ \\
20 & $1.731 \mathrm{e}-08$ & $1.869 \mathrm{e}-06$ & $1.268 \mathrm{e}-12$ & $9.786 \mathrm{e}-10$ & $3.049 \mathrm{e}-05$ \\
\hline
\end{tabular}

Table 7: maximum error of the standard Lane-Emden equations for $m=0,1,5$ respectively.

\begin{tabular}{llll}
\hline $\mathrm{n}$ & $\mathrm{m}=0$ & $\mathrm{~m}=1$ & $\mathrm{~m}=5$ \\
\hline 5 & $2.70199 \mathrm{e}-01$ & $7.87765 \mathrm{e}-02$ & $3.54822 \mathrm{e}-01$ \\
10 & $6.90811 \mathrm{e}-03$ & $2.30291 \mathrm{e}-03$ & $6.61006 \mathrm{e}-03$ \\
15 & $1.15061 \mathrm{e}-03$ & $2.87683 \mathrm{e}-04$ & $9.08909 \mathrm{e}-04$ \\
20 & $2.41206 \mathrm{e}-04$ & $4.78916 \mathrm{e}-05$ & $1.71788 \mathrm{e}-05$ \\
\hline
\end{tabular}


of gas acting under the mutual attracting of its molecules and subject to the classical laws of thermodynamics(Davis, 1962; Shawagfeh, 1993).

$$
y^{\prime \prime}(x)+\frac{2}{x} y^{\prime}(x)+y^{m}(x)=0, x \geq 0,
$$

subject to the boundary conditions

$$
y(0)=1, \quad y^{\prime}(0)=0,
$$

where $m \geq 0$ is constant. Substituting $m=0,1,5$ into Eq. (16) leads to the exact solution

$$
\begin{aligned}
& y(x)=1-\frac{1}{3 !} x^{2}, \\
& y(x)=\frac{\sin (x)}{x}, \\
& y(x)=\left(1+\frac{x^{2}}{3}\right)^{-\frac{1}{2}},
\end{aligned}
$$

respectively.

In other cases there aren't any analytic exact solutions. Therefore, we apply ICSRBF method to solve the standard Lane-Emden Eq. (16) for $m=0,1,1.5,2,2.5,3,4$ and 5. To this way now we can construct the residual functions as follows:

$$
\operatorname{Res}(x)=x y_{n}^{(2)}(x)+2\left(y_{n}^{(1)}(x)\right)+x\left(y_{n}(x)\right)^{m},
$$

As said before, to obtain the coefficient $\xi_{i} s, \operatorname{Res}(x)$ is equalized to zero at $N$ points by (11) :

$$
\operatorname{Res}\left(x_{j}\right)=0, \quad j=0,1,2, \ldots, N .
$$

By solving this set of equations, we can find the approximating function $y_{n}(x)$.

Table 2 shows the comparison of the first zeros of standard Lane-Emden equations, from the present method and exact given by Horedt(Horedt, 2004) for $m=0,1,1.5,2,2.5,3$ and 4, respectively.

Table 3, 4, 5 shows the approximation of $y(x)$ for the standard Lane-Emden equation for $m=$ $2,3,4$ respectively obtained by the method proposed in this paper and those obtained by Horedt(Horedt, 2004).

Table 6 represents the $\|$ Res $\|_{2}$ by the present method for $m=1.5,2,2.5,3,4$ for several points and Table 7 represent the maximum error by the present method for $m=0,1,5$ for several points to how that the new method has appropriate convergence rate. The result graph of the standard Lane-Emden equation for $m=0,1,1.5,2,2.5,3,4$ and 5 is shown in Fig. 1.

\subsection{Example 2 (The isothermal gas sphere equation)}

For $p(x)=1, q(y(x))=e^{y(x)}, h(x)=0, \alpha=2, A=0$ and $B=0$, Eq.(13) is the isothermal gas sphere equation

$$
y^{\prime \prime}(x)+\frac{2}{x} y^{\prime}(x)+e^{y(x)}=0, x \geq 0,
$$




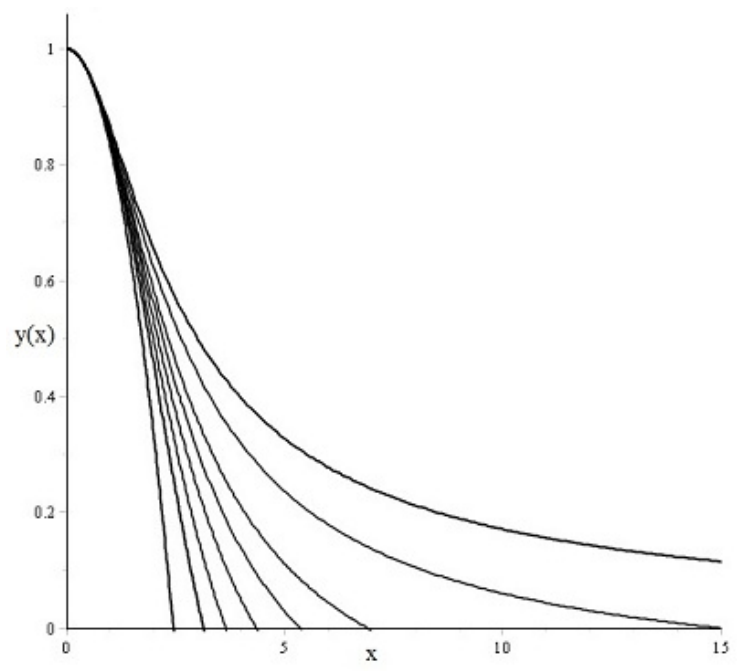

Figure 1: Graph of standard Lane-Emden equation for $\mathrm{m}=0,1,1.5,2,2.5,3,4$ and 5.

Table 8: Comparison of $y(x)$, between present method and series solution given by Wazwaz(Wazwaz, 2001) and HFC method(Parand et al., 2010a) for isothermal gas sphere.

\begin{tabular}{llll}
\hline $\mathrm{x}$ & ICSRBF & HFC & Adomain \\
\hline 0.00 & 0.0000000000 & 0.0000000000 & 0.0000000000 \\
0.1 & -0.0016658338 & -0.0016664188 & -0.0016658339 \\
0.2 & -0.006533671 & -0.0066539713 & -0.0066533671 \\
0.5 & -0.0411539573 & -0.0411545150 & -0.0411539568 \\
1.0 & -0.1588276775 & -0.1588281737 & -0.1588273537 \\
1.5 & -0.3380194248 & -0.3380198308 & -0.3380131103 \\
2.0 & -0.5598230044 & -0.5598233120 & -0.5599626601 \\
2.5 & -0.8063408707 & -0.8063410846 & -0.8100196713 \\
\hline
\end{tabular}




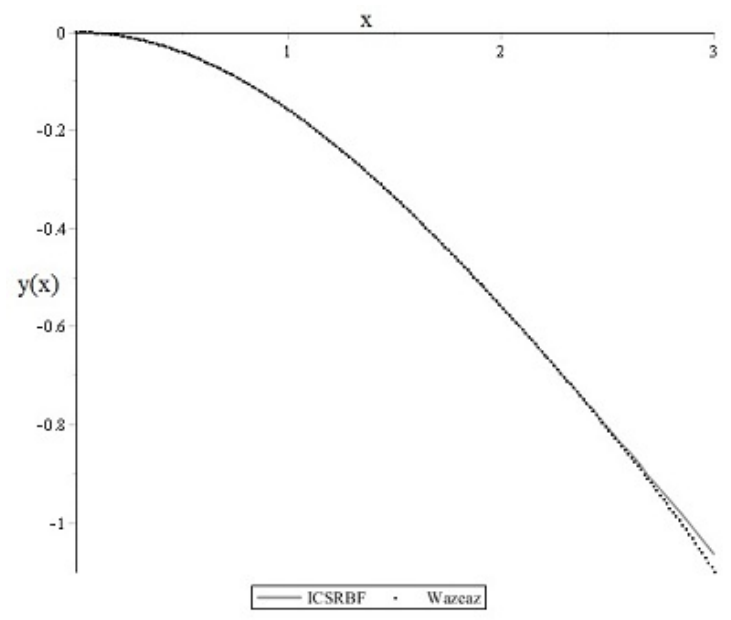

Figure 2: Graph of Isothermal gas sphere equation in comparison with Wazwaz solution(Wazwaz, 2001)

subject to the boundary conditions

$$
y(0)=0, \quad y^{\prime}(0)=0,
$$

Wazwaz(Wazwaz, 2001) by using ADM has produced a series solution as follow:

$$
y(x) \simeq-\frac{1}{6} x^{2}+\frac{2}{5.4 !} x^{4}-\frac{8}{21.6 !} x^{6}+\frac{122}{81.8 !} x^{8}-\frac{61.67}{495.10 !} x^{10} .
$$

We applied the ICSRBF method to solve this equation (18). We construct the residual function as follows:

$$
\operatorname{Res}(x)=x y_{n}^{(2)}(x)+2\left(y_{n}^{(1)}(x)\right)+x e^{\left(y_{n}(x)\right)},
$$

To obtain the coefficients $\xi_{i} s, \operatorname{Res}(x)$ is equalized to zero at $N$ point by (11) with $\gamma=1.7$ :

$$
\operatorname{Res}\left(x_{j}\right)=0, \quad j=0,1,2, \ldots, N .
$$

By solving this set of equations, we have the approximating function $y(x)$. Table 8 shows the comparison of $y(x)$ obtained by method proposed in this paper with $\left(N=40, r_{\omega}=6.5\right)$, and those obtained by Wazwaz(Wazwaz, 2001) (19) and HFC method by Parand et al(Parand et al., 2010a).

In order to compare the present method with those obtained by Wazwaz(Wazwaz, 2001) the resulting graph of Eq.(22) is shown in Fig. 2. The graph of residual for this equation for $N=$ $5,10,15,20,25,30,35$ and 40 is shown in Fig. 3.

\subsection{Example 3 (The White Dwarf equation)}

For $p(x)=1, q(y(x))=\left(y^{2}-\sigma\right)^{\frac{3}{2}}, h(x)=0, \alpha=2, A=1$ and $B=0$, Eq.(13) will be one of the white Dwarf equation that is absorbing to solve

$$
\begin{gathered}
y^{\prime \prime}(x)+\frac{2}{x} y^{\prime}(x)+\left(y(x)^{2}-\sigma\right)^{\frac{3}{2}}=0, x \geq 0, \\
14
\end{gathered}
$$


Table 9: Comparison of $y(x)$, between present method and series solution given by Singh(Singh et al., 2009) and Haar $\operatorname{method}($ Kaur et al., 2013) for White Dwarf equation.

\begin{tabular}{lllll}
\hline $\mathrm{x}$ & $\sigma$ & ICSRBF & MHAM & Haar \\
\hline 0.0001 & 0.1 & 0.999999 & 0.999999 & 1 \\
0.01 & & 0.999985 & 0.999985 & 0.999986 \\
0.1 & & 0.998579 & 0.998578 & 0.998581 \\
0.2 & & 0.994340 & 0.994340 & 0.994379 \\
0.4 & & 0.977738 & 0.977738 & 0.978345 \\
0.6 & & 0.951270 & 0.951263 & 0.953273 \\
0.7 & & 0.934823 & 0.934801 & 0.936497 \\
0.9 & & 0.896673 & 0.896522 & 0.895488 \\
0.0001 & 0.2 & 0.999999 & 0.999999 & 1 \\
0.01 & & 0.999988 & 0.999988 & 0.999988 \\
0.1 & & 0.998809 & 0.998809 & 0.998580 \\
0.2 & & 0.995255 & 0.995251 & 0.995296 \\
0.4 & & 0.981320 & 0.981320 & 0.981931 \\
0.6 & & 0.959049 & 0.959045 & 0.961128 \\
0.7 & & 0.945179 & 0.945165 & 0.947116 \\
0.9 & & 0.912913 & 0.912812 & 0.912833 \\
0.0001 & 0.3 & 0.999999 & 0.999999 & 1 \\
0.01 & & 0.999990 & 0.999990 & 0.999999 \\
0.1 & & 0.999025 & 0.999025 & 0.999028 \\
0.2 & & 0.996115 & 0.996115 & 0.996154 \\
0.4 & & 0.984690 & 0.984690 & 0.985295 \\
0.6 & & 0.966384 & 0.966381 & 0.968464 \\
0.7 & & 0.954953 & 0.954944 & 0.956942 \\
0.9 & & 0.928280 & 0.928216 & 0.928599 \\
\hline
\end{tabular}




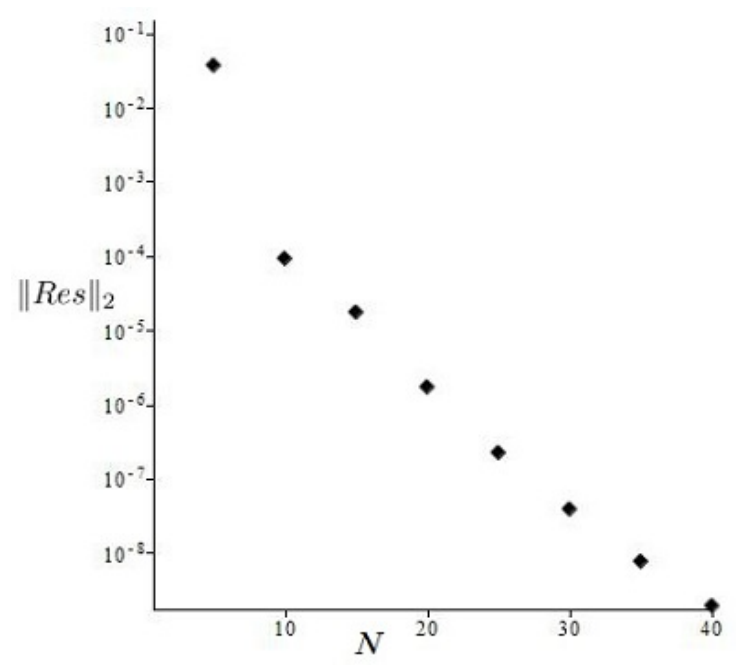

Figure 3: The $\|\operatorname{Res}\|_{2}$ of Isothermal gas sphere equation in for $\mathrm{N}=5,10,15,20,25,30,35$ and 40

subject to the boundry conditions

$$
y(0)=1, \quad y^{\prime}(0)=0,
$$

which was introduced by Chandraskhar (Chandrasekhar, 1967) in his study of the gravitational potential of the degenerate White Dwarf stars. A series solution obtained by Singh(Singh et al., 2009) by using MHAM as follows:

$$
y(x) \simeq 1-\frac{1}{6} \varpi^{3} x^{2}+\frac{1}{40} \varpi^{4} x^{4}-\frac{1}{7 !} \varpi^{5}\left[5 \varpi^{2}+14\right] x^{6} .
$$

where $\varpi=\sqrt{1-\sigma}$.

We applied the ICSRBF method to solve this equation (21). We construct the residual function as follows:

$$
\operatorname{Res}(x)=x y_{n}^{(2)}(x)+2\left(y_{n}^{(1)}(x)\right)+x\left(\left(y_{n}(x)\right)^{2}-\sigma^{2}\right)^{\frac{3}{2}},
$$

To obtain the coefficients $\xi_{i} s, \operatorname{Res}(x)$ is equalized to zero at $N$ point by (11) with $\gamma=1.5$ :

$$
\operatorname{Res}\left(x_{j}\right)=0, \quad j=0,1,2, \ldots, N .
$$

By solving this set of equations,we have the approximating function $y(x)$. Table 9 shows the comparison of $y(x)$ obtained by method proposed in this paper with $\left(N=20, r_{\omega}=0.5\right)$, and those obtained by singh(Singh et al., 2009) (22) and Haar method by Kaur(Kaur et al., 2013).

The result graph of the White Dwarf equation for $\sigma=0.1,0.2$ and 0.3 is shown in Fig. 4. 


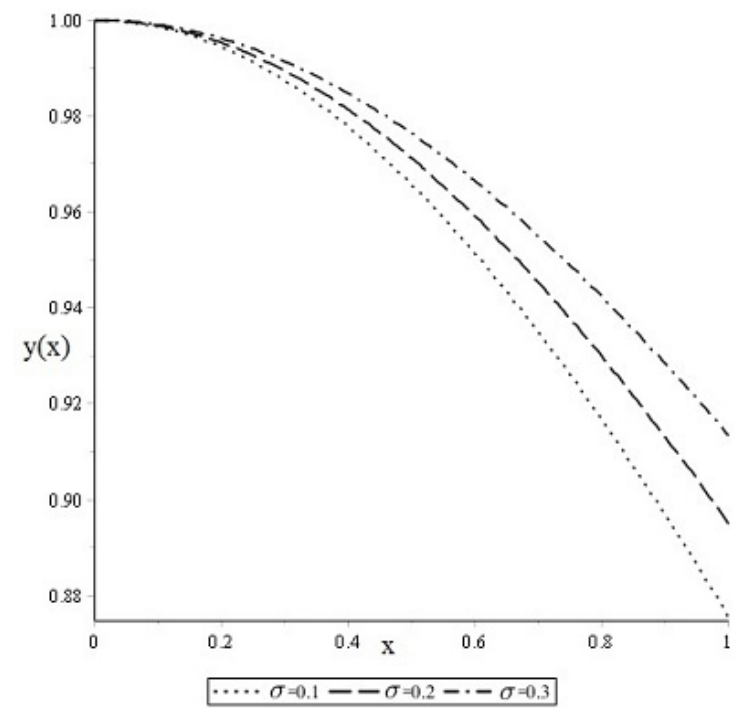

Figure 4: Graph of White Dwarf equation for $\sigma=0.1,0.2$ and 0.3

Table 10: Comparison of $y(x)$, between present method and series solution given by Wazwaz(Wazwaz, 2001) and HFC method(Parand et al., 2010a) for Example 4.

\begin{tabular}{llll}
\hline $\mathrm{x}$ & ICSRBF & HFC & Adomain \\
\hline 0.00 & 1.0000000000 & 1.0000000000 & 1.0000000000 \\
0.1 & 0.9980430038 & 0.9981138095 & 0.9980428414 \\
0.2 & 0.9921896287 & 0.9922758837 & 0.9921894348 \\
0.5 & 0.9519612468 & 0.9520376245 & 0.9519611019 \\
1.0 & 0.8182430031 & 0.8183047481 & 0.8182516669 \\
1.5 & 0.6254386159 & 0.6254886192 & 0.6258916077 \\
2.0 & 0.4066218732 & 0.4066479695 & 0.4136691039 \\
\hline
\end{tabular}




\subsection{Example 4}

For $p(x)=1, q(y(x))=\sinh (y(x)), h(x)=0, \alpha=2, A=1$ and $B=0$, Eq.(13) will be one of the Lane-Emden type equations that is absorbing to solve

$$
y^{\prime \prime}(x)+\frac{2}{x} y^{\prime}(x)+\sinh (y)=0, x \geq 0,
$$

subject to the boundry conditions

$$
y(0)=1, \quad y^{\prime}(0)=0,
$$

A series solution obtained by Wazwaz(Wazwaz, 2001) by using ADM is:

$$
\begin{aligned}
& y(x) \simeq 1-\frac{\left(e^{2}-1\right) x^{2}}{12 e}+\frac{1}{480} \frac{\left(e^{4}-1\right) x^{4}}{e^{2}}- \\
& \frac{1}{30240} \frac{\left(2 e^{6}+3 e^{2}-3 e^{4}-2\right) x^{6}}{e^{3}}+ \\
& \frac{1}{26127360} \frac{\left(16 e^{8}-104 e^{6}+104 e^{2}-61\right) x^{8}}{e^{4}} .
\end{aligned}
$$

We applied the ICSRBF method to solve this equation (24). We construct the residual function as follows:

$$
\operatorname{Res}(x)=x y_{n}^{(2)}(x)+2\left(y_{n}^{(1)}(x)\right)+x \sinh \left(y_{n}(x)\right),
$$

To obtain the coefficients $\xi_{i} s, \operatorname{Res}(x)$ is equalized to zero at $N$ point by (11) with $\gamma=1.7$ :

$$
\operatorname{Res}\left(x_{j}\right)=0, \quad j=0,1,2, \ldots, N .
$$

By solving this set of equations, we have the approximating function $y(x)$. Table 10 shows the comparison of $y(x)$ obtained by method proposed in this paper with $\left(N=20, r_{\omega}=1\right)$, and those obtained by Wazwaz(Wazwaz, 2001) (25) and HFC method by Parand et al.(Parand et al., 2010a).

In order to compare the present method with those obtained by Wazwaz(Wazwaz, 2001) the resulting graph of Eq.(22) is shown in Fig. 5. The graph of $\|\operatorname{Res}\|_{2}$ for this equation for $N=$ 5, 6, 7, 8, 9, 10, 13, 16 and 20 is shown in Fig. 6.

\subsection{Example 5}

For $p(x)=1, q(y(x))=\sin (y(x)), h(x)=0, \alpha=2, A=1$ and $B=0$, Eq.(13) will be one of the Lane-Emden type equations that is absorbing to solve

$$
y^{\prime \prime}(x)+\frac{2}{x} y^{\prime}(x)+\sin (y)=0, x \geq 0,
$$

subject to the boundry conditions

$$
y(0)=1, \quad y^{\prime}(0)=0,
$$




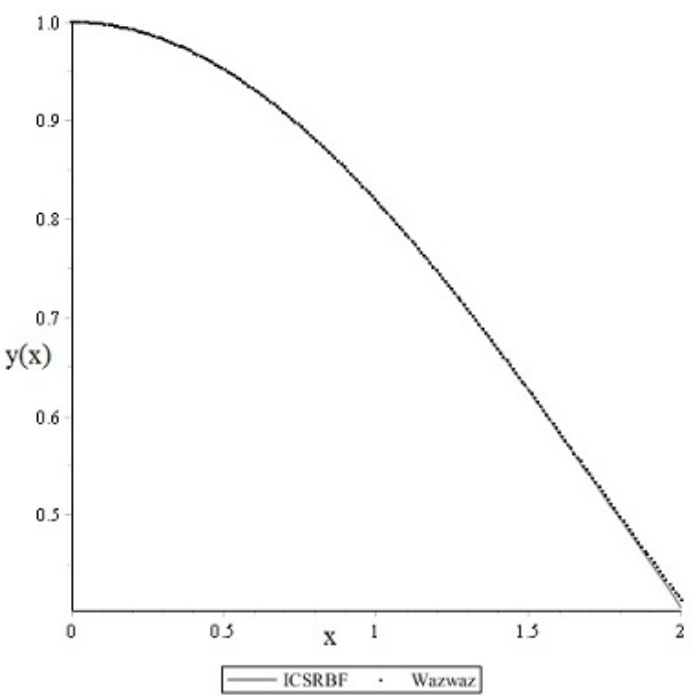

Figure 5: Graph of equation of example 4 in comparing the present method and Wazwaz solution (Wazwaz, 2001).

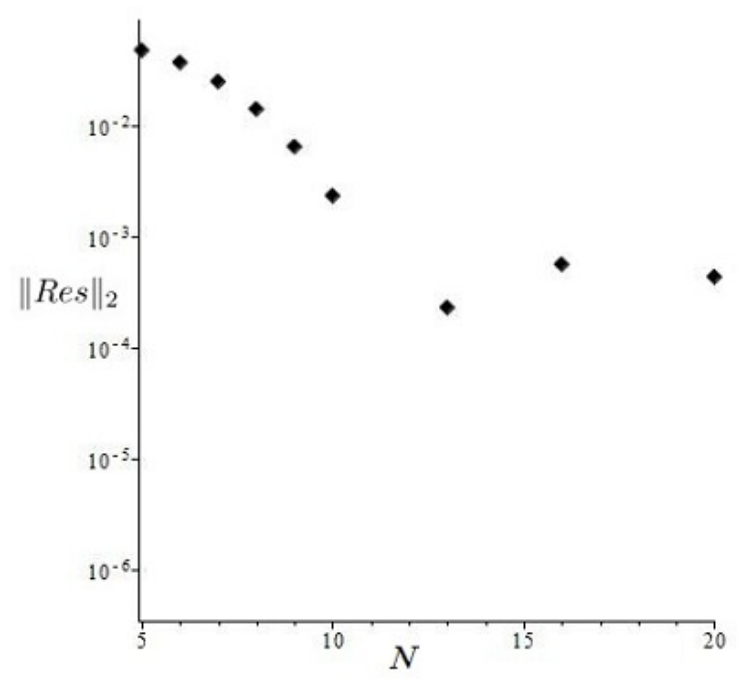

Figure 6: The $\|$ Res $\|_{2}$ of example 4 for $N=5,6,7,8,9,10,13,16$ and 20 
Table 11: Comparison of $y(x)$, between present method and series solution given by Wazwaz(Wazwaz, 2001) and HFC method(Parand et al., 2010a) for Example 5.

\begin{tabular}{llll}
\hline $\mathrm{x}$ & ICSRBF & HFC & Adomian \\
\hline 0.0 & 1.0000000000 & 1.0000000000 & 1.0000000000 \\
0.1 & 0.9985979436 & 0.9986051425 & 0.9985979358 \\
0.2 & 0.9943962892 & 0.9944062706 & 0.9943962733 \\
0.5 & 0.9651778048 & 0.9651881683 & 0.9651777886 \\
1.0 & 0.8636811302 & 0.8636881301 & 0.8636811027 \\
1.5 & 0.7050451897 & 0.7050524103 & 0.7050419247 \\
2.0 & 0.5064632371 & 0.5064687568 & 0.5063720330 \\
\hline
\end{tabular}

A series solution obtained by Wazwaz(Wazwaz, 2001) by using ADM is:

$y(x) \simeq 1-\frac{1}{6} k_{1} x^{2}+\frac{1}{120} k_{1} k_{2} x^{4}+k_{1}\left(\frac{1}{3024} k_{1}^{2} \cdot-\frac{1}{5040} k_{2}^{2}\right) x^{6}+k_{1} k_{2}\left(-\frac{113}{3265920} k_{1}^{2}+\frac{1}{362880} k_{2}^{2}\right) x^{8}+\ldots$

where $k_{1}=\sin (1)$ and $k_{2}=\cos (1)$.

We applied the ICSRBF method to solve this equation (27). We construct the residual function as follows:

$$
\operatorname{Res}(x)=x y_{n}^{(2)}(x)+2\left(y_{n}^{(1)}(x)\right)+x \sin \left(y_{n}(x)\right),
$$

To obtain the coefficients $\xi_{i} s, \operatorname{Res}(x)$ is equalized to zero at $N$ point by (11) with $\gamma=1.6$ :

$$
\operatorname{Res}\left(x_{j}\right)=0, \quad j=0,1,2, \ldots, N .
$$

By solving this set of equations, we have the approximating function $y(x)$. Table 11 shows the comparison of $y(x)$ obtained by method proposed in this paper with $\left(N=20, r_{\omega}=2\right)$, and those obtained by Wazwaz(Wazwaz, 2001) (28) and HFC method by Parand et al. (Parand et al., 2010a).

In order to compare the present method with those obtained by Wazwaz(Wazwaz, 2001) the resulting graph of Eq.(27) is shown in Fig. 7.

The graph of $\|\operatorname{Res}\|_{2}$ for this equation for $N=5,6,7,8,9,10,13,16$ and 20 is shown in Fig. 8.

\section{Conclusion}

Lane-Emden equations occur in the theory of stellar structure and describe the temperature variation of a spherical gas cloud. White Dwarf equation appears in the gravitational potential of the degenerate White Dwarf stars. Lane-Emden type equations have been considered by many mathematicians as mentioned before(Dehghan and Shakeri, 2008a). The fundamental goal of this paper has been to construct an approximation to the solution of non-linear Lane-Emden type equation in a semi-infinite interval. CSRBFs are proposed to provide an effective but simple way to improve the convergence of the solution by the collocation method. The validity of the method is based on the assumption that it converges by increasing the number of collocation points. A comparison is made among the numerical solution of Horedt(Horedt, 2004) and series solutions 


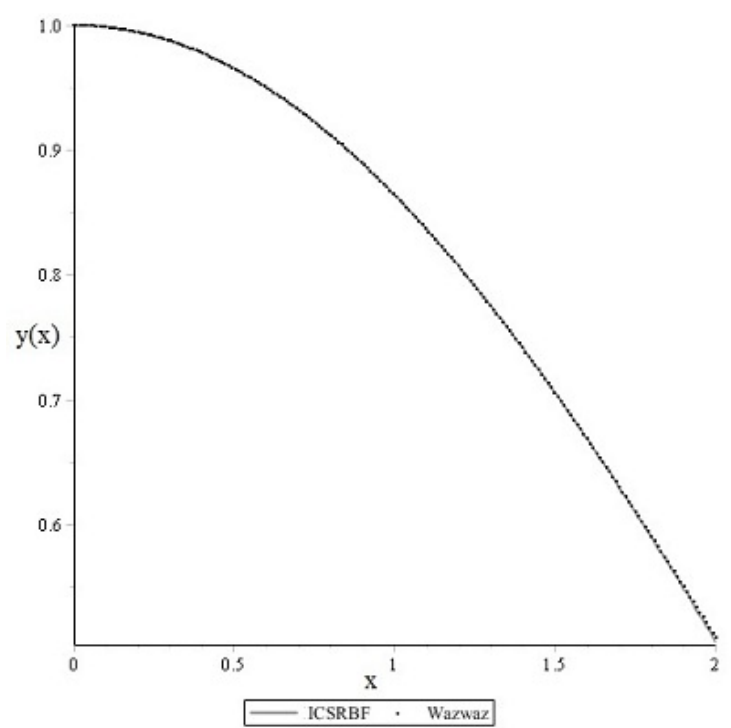

Figure 7: Graph of equation of example 5 in comparing the present method and Wazwaz solution (Wazwaz, 2001).

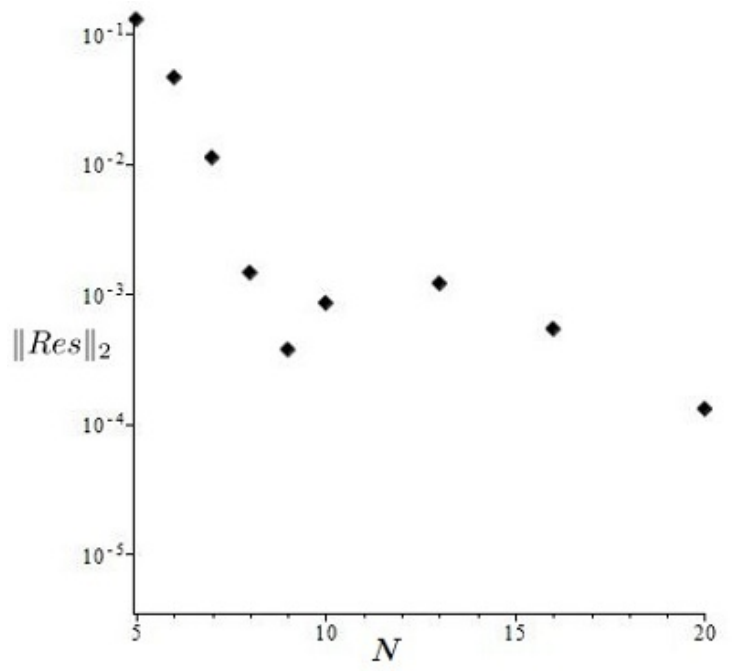

Figure 8: The $\|\operatorname{Res}\|_{2}$ graph of example 5 for $N=5,6,7,8,9,10,13,16$ and 20 
of Wazwaz(Wazwaz, 2001), Singh et al.(Singh et al., 2009), Kaur et al.(Kaur et al., 2013) and the current work. It has been shown that our present work provides an acceptable approach for the Lane-Emden type equations. Also it was confirmed by maximum error and $\|$ Res $\|_{2}$ figures, this approach has an exponentially convergence rate. Also, in this paper we show that the ICSRBF method for solving ordinary differential equations is simple and it has high accuracy and reliable convergence.

\section{References}

Abbasbandy, S., Azarnavid, B., Hashim, I., Alsaedi, A., 2014. Approximation of backward heat conduction problem using gaussian radial basis functions. U.P.B. Sci. Bull., Series A 76, 67-76.

Adibi, H., Rismani, A. M., 2010. On using modified legenre-spectral method for solving singular ivps of lane-emden type. Comput. Math. Applic. 60, 2126-2130.

Agrawala, P. R., O’Regnab, D., 2007. Appl. Math. Lett. 20, 119812005.

Akyüz-Daşcıoğlu, A., Çerdik Yaslan, H., 2011. The solution of high-order nonlinear ordinary differential equations. Appl. Math. Comput. 217, 5658-5666.

Aslanov, A., 2008. Determination of convergence intervals of the series solution of emden-fowler equations using polytropes and isothermal sphere. Phys. Lett. A 372, 3555-3561.

Azarnavid, B., Parvaneh, F., Abbasbandy, S., 2015. Picard-reproducing kernel hilbert space method for solving generalized singular nonlinear lane-emden type equations. Math. Model. Anal. 20, 754-767.

Bataineh, A. S., Noorani, M. S. M., Hashim, I., 2009. Homotopy analysis method for singular IVPs of Emden-Fowler type. Commun. Nonlinear. Sci. Numer. Simul. 14, 1121-1131.

Bender, C. M., Milton, K. A., Pinsky, S. S., Simmons, L. M., 1989. A new perturbative approach to nonlinear problems. J. Math. Phys. 30, 1447-1455.

Benko, D., Biles, D. C., Robinson, M. P., Sparker, J. S., 2008. Nyström method and singular second-order differentil equations. Comput. Math. Applic. 56, 1975-1980.

Bharwy, A. H., Alofi, A. S., 2012. A jacobi-gauss collocation method for solving nonlinear lane-emden type equations. Commun. Nonl. Sci. Numer. Simul. 17, 62-70.

Boubaker, K., Van-Gorder, R. A., 2012. Application of the bpes to lane-emden equations governing polytropic and isothermal gas sphere. New. Astron. 17, 565-569.

Boyd, J. P., 2011. Chebyshev spectral methods and the lane-emden problem. Numer. Math. Theor. Meth. Appl. 4 (2), $142-157$.

Bu, W., Ting, Y., Wu., Y., Yang, J., 2015. Finite difference/finite element method for two-dimensional space and time fractional blochtorrey equations. J. Comput. Phys. 293, 264-279.

Buhman, M. D., 2000. Radial basis functions. Acta Numerica, 1-38.

Buhman, M. D., 2004. Radial basis functions: Theory and Imolemantations. Cambridge University Press, New York.

Caruntu, B., Bota, C., 2013. Approximate polynomial solutions of the nonlinear lane-emden type equations arising in astrophysics using the squared reminder minimization method. Comput. Phys. Commun. 184, 1643-1648.

Chandrasekhar, S., 1967. Introduction to the study of stellar structure. Dover, New York.

Cheng, A. H. D., Golberg, M. A., Kansa, E. J., Zammito, Q., 2003. Exponential convergence and h-c multiquadric collocation method for partial differential equations. Numer. Meth. Part. D. E. 19, 571-594.

Choi, H. J., Kweon, J. R., 2016. A finite element method for singular solutions of the navierstokes equations on a non-convex polygon. J. Comput. Appl. Math. 292, 342-362.

Chowdhury, M. S. H., Hashim, I., 2009. Solution of Emden-Fowler equations by Homptopy Perturbation method. J. Nonlinear Anal. Ser. A Theor. Method 10, 104-115.

Davis, H. T., 1962. Introduction of Nonlinear Differentail and Integral Equations. Dover, New York.

Dehghan, M., Saadatmandi, A., 2007. The numerical solution of a nonlinear system of second-order boundry value problems using sinc-collocation method. Math. Comput. Model. 46, 1434-1441.

Dehghan, M., Shakeri, F., 2008a. Approximat solution of a differential equation arising in Astrophysics using the VIM. New Astron. 13, 53-59.

Dehghan, M., Shakeri, F., 2008b. Use of he's homotopy perturbation method for solving a partial differential equation arising in modeling of flow in porous media. J. Porous Media 11, 765-778.

Dehghan, M., Shokri, A., 2008. A numerical method for solution of the two-dimensional sine-gordon equation using the radial basis functions. Math. Comput. Simul. 79, 700-715.

Dehghan, M., Shokri, A., 2009a. A meshless method for numerical solution of the one-dimensional wave equation with an integral condition using radial basis functions. Numerical Algorithms 52 (3), 461-477. 
Dehghan, M., Shokri, A., 2009b. A meshless method for numerical solution of the one-dimensional wave equation with an integral condition using radial basis functions. Numer. Algorithms 52, 461-477.

Dehghan, M., Shokri, A., 2009c. Numerical solution of the nonlinear kleingordon equation using radial basis functions. J. Comput. Appl. Math. 230, 400-410.

Doha, E. H., Abd-Elhameed, W. M., Youssri, Y. H., 2013. Second kind chebyshev operational matrix algorithm for solving differential equations of lane-emden type. New. Astro. 23-24, 113-117.

Fasshauer, G. E., 2007. Meshfree Approximation Methods With Matlab. World Scientific Publishing Co. Pte. Ltd

Gürbüz, B., Sezer, M., 2014. Laguerre polynomial approach for solving lane-emden type functional differential equations. Appl. Math. Comput. 242, 255-264.

He, J. H., 2003. Variational approach to the lane-emden equation. Appl. Math. Comput. 143, 539-541.

Horedt, G. P., 2004. Polytropes: applications in Astrophysics and related fileds. Kluwer Academic Publishers, Dordecht

Hosseini, S. G., Abbasbandy, S., 2015. Solution of lane-emden type equations by combination of the spectral method and adomian decomposition method. U.P.B. Sci. Bull., Series A 2015, 1-10.

Iqbal, S., Javad, A., 2011. Application of optimal homotopy asymptotic method for the analytic solution of singular lane-emden type equation. Appl. Math. Comput. 217, 7753-7761.

Islam, S. U., S. Haqb, A. A., 2009. A meshfree method for the numerical solution of the rlw equation. J. Comput. Appl. Math. 223, 997-1012.

Kansa, E. J., 1990a. Multiquadrics-a scratted dat approximation scheme with applications to computational fluiddynamic-i surface approximations ans partial derivative estimates. Comput. Math. Appl. 19, 127-145.

Kansa, E. J., 1990b. Multiquadrics-a scratted dat approximation scheme with applications to computational fluiddynamic-ii solutions to parabolic, hyperbolic and elliptic partial differential equations. Comput. Math. Appl. 19, $147-161$.

Kansa, E. J., Hon, Y. C., 2000. Circumventing the ill-conditioning problem with multiquadric radial basis functions applications to elliptic partial defferential equations. Comput. Math. Appl. 39, 123-137.

Kaur, H., Mittal, R. C., Mishra, V., 2013. Haar wavelet approximate solutions for the generalized lane-emden equations arising in astrophysics. Comput. Phys. Commun. 184, 2169-2177.

Kazemi-Nasab, A., Kılıçman, A., Atabakan, Z. P., Leong, W. J., 2015. A numerical approach for solving singular nonlinear lane-emden type equations arising in astrophysics. New. Astro. 34, 178-186.

Lee, J. C., Hon, Y. C., 2004. Domain decomposition for radial basis meshless method. Numer. Methods Partial Differential Eq. 20, 450-462.

Liao, S., 2003. A new analytic algorithm of lane-emden type equation. Appl. Math. Comput. 142, 1-16.

Mai-Duy, N., 2005. Solving high order ordinary differential equations with radial basis function networks. Int. J. Numer. Meth. Eng. 62, 824-852.

Mai-Duy, N., Tran-Cong, T., 2001a. Numerical solution of differential equations using multiquadric radial basis function networks. Neural Netw 14, 185-199.

Mai-Duy, N., Tran-Cong, T., 2001b. Numerical solution of navier-stocks equations using multiquadric radial basis function networks. Int. J. Numer. Meth. Fl. 37, 65-86.

Mall, S., Chakraverty, S., 2014. Chebyshev neural network based model for solving lane-emden type equations. Appl. Math. Comput. 247, 100-114.

Mendelzweig, V. B., Tabakin, F., 2001. Quasilinearization approach to nonlinear problems in physics with application to nonlinear odes. Comput. Phys. Commun. 141, 268-281.

Nazari-Golshan, A., Nourazar, S. S., Ghafoori-Fard, H., Yıldırım, A., Campo, A., 2013. A modified homotopy perturbation method couled with the fourier transform for nonlinear and singular lane-emden equations. Appl. Math. Lett. 26, 1018-1025.

Noye, B. J., Dehghan, M., 1999. New explicit finite difference schemes for two-dimensional diffusion subject to specification of mass. Numer. Meth. Par. Diff. Eq. 15, 521-534.

Pandey, R. K., Kumar, N., 2012. Solution of lane-emden type equations using bernstein operational matrix of differentation. New. Astro. 17, 303-308.

Pandey, R. K., Kumar, N., Bhardwaj, A., Dutta, G., 2012. Solution of lane-emden type equations using legendre operational matrix of differentation. Appl. Math. Comput. 218, 7629-7637.

Parand, K., Abbasbandy, S., Kazem, S., Rad, J., 2011a. A novel application of radial basis functions for solving a model of first-order integro-ordinary differential equation. Commun. Nonlinear Sci. Numer. Simul. 16, 4250-4258.

Parand, K., Abbasbandy, S., Kazem, S., Rezaei, A., 2011b. An improved numerical method for a class of astrophysics problems based on radial basis functions. Physica Scripta 83 (1), 015-011.

Parand, K., Dehghan, M., Pirkhedri, A., 2013a. The sinc-collocation method for solving the thomas-fermi equation Journal of Computational and Applied Mathematics 237 (1), 244-252.

Parand, K., Dehghan, M., Rezaei, A. R., Ghaderi, S. M., 2010a. An approximation algorthim for the solution of the nonlinear Lane-Emden type equation arising in Astorphisics using Hermit functions Collocation method. Comput. Phys. Commun. 181, 1096-1108. 
Parand, K., Dehghan, M., Taghavi, A., 2010b. Modified generalized laguerre function tau method for solving laminar viscous flow: The blasius equation. International Journal of Numerical Methods for Heat \& Fluid Flow 20 (7), 728743.

Parand, K., Hossayni, S. A., Rad, J., 2016. An operation matrix method based on bernstein polynomials for riccati differential equation and volterra population model. Applied Mathematical Modelling.

Parand, K., Khaleqi, S., 2016. Rational chebyshev of second kind collocation method for solving a class of astrophysics problems. The European Physical Journal - Plus.

Parand, K., Nikarya, M., Rad, J. A., 2013b. Solving non-linear lane-emden type equations using bessel orthogonal functions collocation method. Celest. Mech. Dyn. Astr. 116, 97-107.

Parand, K., Rad, J. A., 2013. Kansa method for the solution of a parabolic equation with an unknown spacewisedependent coefficient subject to an extra measurement. Comp. Phys. Commun. 184, 582-595.

Parand, K., Razzaghi, M., 2004a. Rational chebyshev tau method for solving higher-order ordinary differential equations. Int. J. Comput. Math. 81 (1), 73-80.

Parand, K., Razzaghi, M., 2004b. Rational legendre approximation for solving some physical problems on semi-infinite intervals. Phys. Scr. 69, 353-357.

Rad, J. A., Kazem., S., Parand, K., 2012. A numerical solution of the nonlinear controlled duffing oscillator by radial basis function. Comput. Math. Appl. 64, 2049-2065.

Rad, J. A., Kazem, S., Shaban, M., Parand, K., Yildirim, A., 2014. Numerical solution of fractional differential equations with a tau method based on legendre and bernstein polynomials. Math. Meth. Appl. Sci. 37, 329-342.

Rad, J. A., Parand, K., Abbasbandy, S., 2015a. Local weak form meshless techniques based on the radial point interpolation (rpi) method and local boundary integral equation (lbie) method to evaluate european and american options. Communications in Nonlinear Science and Numerical Simulation 22 (1), 1178-1200.

Rad, J. A., Parand, K., Ballestra, L. V., 2015b. Pricing european and american options by radial basis point interpolation. Applied Mathematics and Computation 251, 363-377.

Ramos, J. I., 2003. Linearization methods in classical and quantum mechanics. Comput. Phys. Commun. 153 (2), 199 208.

Ramos, J. I., 2005. Linearization techniques for singular initial-value problems of ordinary differential equations. J. Appl. Math. Comput. 161, 525-542.

Ramos, J. I., 2007. Piecewise-adaptive decomposition method. Chaos. Solit. Fract. 40 (4), 1623-1636.

Ramos, J. I., 2008. Series approach to the lane-emden equation and comparison with the homotopy perturbation method. Chaos. Solit. Fract. 38 (2), 400-408.

Rashidi, K., Adibi, H., Rad, J. A., Parand, K., 2014. Application of meshfree methods for solving the inverse onedimensional stefan problem,. Eng. Anal. Bound. Elem. 40, 1-21.

Rismani, A. M., Monfared, H., 2012. Numerical solution of singular inps of lane-emden type using a modified legendrespectral method. Appl. Math. Model. 36, 4830-4836.

Sara, S. A., 2005. Adaptive radial basis function method for time dependent partial differential equations. Appl. Numer. Math. 54, 79-94.

Shawagfeh, N. T., 1993. Nonperturbative approximate solution of Lane-Emden equation. J. Math. Phys. 34, 4364-4369.

Shen, Q., 2012. A meshless scaling iterative algorithm based on campactly supported radial basis functions for the numerical solution of lane-emden-fowler equation. Numer. Methods Partial Differential Eq. 28, 554-572.

Shokri, A., Dehghan, M., 2010. A not-a-knot meshless method using radial basis functions and predictorcorrector scheme to the numerical solution of improved boussinesq equation. Comput. Phys. Commun. 181, 1990-2000.

Singh, O. P., Pandey, R. K., Singh, V. K., 2009. An analytic algorithm of Lane-Emden type equations arising in Astropysics using modified Homotopy analysis method. Comput. Phys. Commun. 180, 1116-1124.

Vanani, S. K., Aminataei, A., 2010. On the numerical solution of differential equations of lane-emden type. Comput. Math. Applic. 59, 2815-2820.

Wazwaz, A. M., 2001. A new algorithm for solving differential equations of lane-emden type. Appl. Math. Comput. 118 287-310.

Wazwaz, A. M., 2006. The modified decomposition method for analytic treatment of differential equations. Appl. Math. Comput. 173, 165-176.

Wendland, H., 1995. Piecewise polynomial, positive definite and compactly supported radial functions of minimal degree. Adv. Comput. Math. 4, 389-396.

Wendland, H., 2005. Scattered Data Approximation. Cambridge University Press, New York.

Wong, S. M., Hon, Y. C., Golberg, M. A., 2002. Compactly supported radial basis function for shallow water equations. Appl. Math. Comput. 127, 79-101.

Yıldırım, A., Öziş, T., 2007. Solutions of singular ivps of lane-emden type homotopy perturabation method. Phys. Lett. A 369, 70-76.

Yıldırım, A., Öziş, T., 2009. Solutions of singular IVPs of Lane-Emden type equations by the VIM method. J. Nonlinear Anal. Ser. A Theor. Method 70, 2480-2484. 
Yousefi, S. A., 2006. Legendre Wavlate method for solving differential equations of Lane-Emden type. Appl. Math. Comput 181, 1417-1422.

Yüzbaşı, S., 2011. A numerical approach for solving the high-order linear singular differential-difference equations. Comput. Math. Applic. 62, 2289-2303.

Yüzbaşı, S., Sezer, M., 2013. An improved bessel collocation method with a residual error function to solve a class of lane-emden differential equations. Math. Comput. Model. 57, 1298-1311. 